Draft Version November 15, 2018

Preprint typeset using $\mathrm{IAT}_{\mathrm{E}} \mathrm{X}$ style emulateapj v. 5/2/11

\title{
THE ABSENCE OF COLD DUST AROUND WARM DEBRIS DISK STAR HD 15407A
}

\author{
Hideaki Fujiwara $^{1}$, TAKashi Onaka ${ }^{2}$, Satoshi Takita ${ }^{3}$, TakuYa Yamashita ${ }^{4}$, Misato Fukagawa ${ }^{5}$, \\ Daisuke Ishihara ${ }^{6}$, Hirokazu KatazA ${ }^{3}$, and Hiroshi Murakami ${ }^{3}$ \\ Draft version November 15, 2018
}

\begin{abstract}
We report Herschel and $A K A R I$ photometric observations at far-infrared (FIR) wavelengths of the debris disk around the F3V star HD 15407A, in which the presence of an extremely large amount of warm dust ( 500-600 K) has been suggested by mid-infrared (MIR) photometry and spectroscopy. The observed flux densities of the debris disk at 60-160 $\mu \mathrm{m}$ are clearly above the photospheric level of the star, suggesting excess emission at FIR as well as at MIR wavelengths previously reported. The observed FIR excess emission is consistent with the continuum level extrapolated from the MIR excess, suggesting that it originates in the inner warm debris dust and cold dust $(\sim 50-130 \mathrm{~K})$ is absent in the outer region of the disk. The absence of cold dust does not support a late heavy bombardment-like event as an origin of the large amount of warm debris dust around HD 15047A.
\end{abstract}

Subject headings: circumstellar matter — zodiacal dust — infrared: stars — stars: individual (HD 15407A)

\section{INTRODUCTION}

Debris disks are considered as dusty disks replenished by the formation of the second generation dust during the main-sequence star phase, not as direct leftovers of protoplanetary disks (e.g. Backman \& Paresce 1993). Therefore debris disks hold hints of circumstellar rocky materials such as planets or their building blocks. Recent mid-infrared (MIR) observations by Spitzer and AKARI of high sensitivity have revealed the presence of warm debris disks that show MIR excess emission over the photosphere (e.g. Fujiwara et al. 2010). Some warm debris disks possess a large amount of dust and cannot be explained by steady-state evolution of the disks by planetesimal collisions. They may be formed by transient events (Wvatt et al. 2007). As transient events that generate bright warm debris disks, two major mechanisms are suggested. One is akin to the late heavy bombardment (LHB), which is suggested to account for the debris disk around $\eta$ Corvi (Lisse et al. 2012). The other is a two-body impact, which is akin to the giant impact that created the Moon, and suggested to account for the debris disk around BD +20 307 (Weinberger et al. 2011). Examination of transient events relevant to debris disks is important not only for the understanding of dynamical evolution and presence of small bodies in the planetary system, but also for the understanding of the history of our solar system.

$\mathrm{HD}$ 15407A is an F3V main-sequence star at the

\footnotetext{
${ }^{1}$ Subaru Telescope, National Astronomical Observatory of Japan, 650 North A'ohoku Pl., Hilo, HI 96720, USA; hideaki@naoj.org

${ }^{2}$ Department of Astronomy, School of Science, University of Tokyo, Bunkyo-ku, Tokyo 113-0033, Japan

3 Institute of Space and Astronautical Science, Japan Aerospace Exploration Agency, 3-1-1 Yoshinodai, Chuo-ku, Sagamihara, Kanagawa 252-5210, Japan

${ }_{4}^{4}$ National Astronomical Observatory of Japan, 2-21-1 Osawa, Mitaka, Tokyo 181-0015, Japan

${ }^{5}$ Graduate School of Science, Osaka University, 1-1 Machikaneyama, Toyonaka, Osaka 560-0043, Japan

${ }^{6}$ Department of Physics, School of Science, Nagoya University, Furo-cho, Chikusa-ku, Nagoya, Aichi 464-8602, Japan
}

distance of $55 \mathrm{pc}$ from the Sun (van Leeuwen 2007), which possesses one of the most extreme warm debris disks. Large excess over the expected stellar photospheric emission at $\lambda \gtrsim 5 \mu \mathrm{m}$ has been reported by IRAS, AKARI, and Spitzer observations (Oudmaijer et al. 1992; Fujiwara et al. 2012a). The fractional luminosity (fraction of excess luminosity over stellar luminosity) of the MIR excess emission is estimated as $L_{\text {dust }} / L_{*} \sim 0.005$. Fine dust of silica $\left(\mathrm{SiO}_{2}\right)$ and amorphous silicate are detected toward the star (Fuiiwara et al. 2012a). The effective temperature and the metallicity of the star have been estimated as $6350 \mathrm{~K}$ and $[\mathrm{Fe} / \mathrm{H}]=+0.08$, respectively, by GenevaCopenhagen Survey (Holmberg et al. 2009) and highdispersion spectra obtained with Okayama Astrophysical Observatory/HIDES (Fujiwara et al. 2012a). As for the stellar age, two values, $80_{-20}^{+40} \mathrm{Myr}$ (Melis et al. 2010) and $2.1 \pm 0.3$ Gyr (Holmberg et al. 2009), have been suggested.

In this Letter, we report new far-infrared (FIR) photometry of HD 15407A obtained with Herschel and AKARI, which constrains the amount of lowtemperature dust around the star. We report the detection of FIR excess emission toward the star, which is accounted for solely by the MIR-emitting warm dust, suggesting the absence of cold dust around the star. We discuss the possible formation mechanism of the warm debris disk around HD 15407A.

\section{OBSERVATIONS AND DATA REDUCTION \\ 2.1. Herschel/PACS Observations}

HD 15407A was observed on 2011 July 15 (UT) with Photodetector Array Camera and Spectrometer (PACS; Poglitsch et al. 2010) onboard Herschel (Pilbratt et al. 2010) using the photometer (scan map) mode (Observation ID: 1342224222-1342224225, PI: Ben Zuckerman) with the three channels: $70 \mu \mathrm{m}$ (blue), $100 \mu \mathrm{m}$ (green), and $160 \mu \mathrm{m}$ (red). We use the pipeline-processed Level-2 data products taken from the Herschel Science Archive. The star is seen clearly in the blue and green channel im- 
ages and marginally in the red channel images. A very red source is also seen at the separation of $30^{\prime \prime}$ north of HD 15407A in the green and red images.

Aperture photometry is conducted for all the individual images using IRAF apphot task. The aperture radius and sky annulus dimensions are chosen as $5^{\prime \prime}$ and $50^{\prime \prime}-70^{\prime \prime}$, respectively, to obtain the best signal-to-noise ratios and the minimum contamination by the nearby source. Aperture correction is applied to the measured flux density using the aperture correction factors for a $5^{\prime \prime}$ aperture shown in PACS Observer's Manual (2011). We calculate the weighted mean of the flux density of individual observations for each band. We take the $3 \sigma$ of scatter of individual measurements as the photometric uncertainty for each band. The derived flux densities are shown in Table 1. Color correction is not applied. The color-correction factors for sources of $\gtrsim 50 \mathrm{~K}$ are 0.98-1.07 (Poglitsch et al. 2010) and much less than the photometric errors.

\subsection{AKARI/FIS Slow-scan Observations}

HD 15407A was observed on 2007 August 18 (UT) with the Far-Infrared Surveyor (FIS; Kawada et al. 2007) onboard AKARI using the FIS01 observation mode as part of the director's time (Observation ID: 5110122-001). The FIS was operated in the photometry mode with the four bands: N60 $(65 \mu \mathrm{m})$, WIDE-S $(90 \mu \mathrm{m})$, WIDE$L(140 \mu \mathrm{m})$, and $N 160(160 \mu \mathrm{m})$. The FIS data were processed with the FAST reduction toolkit for FIS slowscan observation data, which is distributed within the $A K A R I$ internal team at present. Basic reduction procedure is the same as the FIS Slow-Scan Toolkit Version 20070914 officially provided. Among the four-band images, a source is seen at the position of HD 15407A only in the N60 and WIDE-S images. The detected source is significantly affected by the dead pixel in the N60 image and reliable photometry of the source is difficult for this band.

Aperture photometry is conducted only for the WIDE$S$ images using IRAF apphot task. The aperture radius and sky annulus dimensions are chosen as $40^{\prime \prime}$ and $120^{\prime \prime}$ $200^{\prime \prime}$, respectively. Aperture correction and flux calibration for point source are applied to the measured flux density as described in Shirahata et al. (2009). Due to the large beam size of $A K A R I /$ FIS (30" for WIDE-S), the nearby source detected by Herschel/PACS contaminates the signal. Since Herschel/PACS observations suggest that the signal count of the nearby source is about half of HD 15407A at $\sim 100 \mu \mathrm{m}$, the measured flux density in the $W I D E-S$ band is divided by 1.5 to estimate the flux density of HD 15407A. The final estimated flux density of HD 15407A in the WIDE-S band of $A K A R I /$ FIS is shown in Table 1

\subsection{Data from Literature and Catalog: WISE, AKARI/IRC, IRAS, Subaru/COMICS, Spitzer/IRS, 2MASS}

In addition to the FIR photometry described above, we collect available infrared photometric data from literature and catalogs. Near-infrared (NIR) $J H K_{\mathrm{S}}$ photometry is taken from Two Micron All Sky Survey (2MASS) Point Source Catalog (Cutri et al. 2003). NIR to MIR magnitudes at 3.4 (W1 band), 4.6 (W2 band), 12 (W3 band), and $22 \mu \mathrm{m}$ (W4 band) are taken from WideField Infrared Survey Explorer (WISE) All-Sky Data Release (Cutri et al. 2012). Broad-band MIR photometric data at 9 ( $S 9 \mathrm{~W}$ band) and $18 \mu \mathrm{m}$ ( $L 18 \mathrm{~W}$ band) are taken from the AKARI/IRC All-Sky Survey Point Source Catalog (Ishihara et al. 2010). Narrowband MIR photometric data with higher spatial resolution at 8.8, 11.7, and $18.8 \mu \mathrm{m}$ are also taken with Subaru/COMICS (Fuijwara et al. 2012b). IRAS photometric data in the MIR and FIR are taken from Point Source Catalog with the upper limits at 60 and $100 \mu \mathrm{m}$ (Beichman et al. 1988). All the collected flux densities are shown in Table 1.

\section{RESULTS}

\subsection{Spectral Energy Distribution and FIR Excess - Absence of Cold Dust}

The obtained spectral energy distribution (SED) of HD 15407A compiled with all the measured and collected photometric data in the NIR-FIR is shown in Figure1. We also plot the Spitzer/IRS spectrum at 5-35 $\mu \mathrm{m}$ and the photospheric emission of HD 15407A estimated from the Kurucz model (Kurucz 1992) with the effective temperature of $6500 \mathrm{~K}$ and the surface gravity of $\log g=+4.0$ fitted to the 2MASS $K_{\mathrm{S}}$-band photometry (Fujiwara et al. 2012a). Significant excess emission over the photosphere in the FIR is clearly shown by the Herschel/PACS and AKARI/FIS photometry, in addition to the large MIR excess previously reported. The detected flux densities at $70-160 \mu \mathrm{m}$ are $\gtrsim 10$ times larger than the photospheric emission.

In Figure 1, we plot the continuum level of the excess emission (a blackbody of the temperature $T=505 \mathrm{~K}$ with the fractional luminosity $\left.L_{\text {dust }} / L_{*}=0.005\right)$ estimated from the dust model fitted to the Spitzer/IRS spectrum at 5-35 $\mu \mathrm{m}$ (Fuijwara et al. 2012a). The Herschel/PACS flux densities at 70 and $100 \mu \mathrm{m}$ are consistent with the extrapolation of the estimated continuum level of the MIR excess within a $1 \sigma$ photometric uncertainty, suggesting that the excess at 70 and $100 \mu \mathrm{m}$ originates in the inner warm debris dust. $A K A R I /$ FIS photometry at $90 \mu \mathrm{m}$ is also consistent with the estimated continuum level within a $1 \sigma$ uncertainty. The consistency between the continuum estimated from the Spitzer/IRS spectrum $(5-35 \mu \mathrm{m})$ and the FIR photometry $(70-100 \mu \mathrm{m})$ suggests the absence of cold materials of $\sim 130-50 \mathrm{~K}$ in the outer region $\left(R_{\text {dust }}=10-60 \mathrm{AU}\right)$ of the HD 15407A system since such cold materials should show additional emission peaking at $35-100 \mu \mathrm{m}$ over the continuum, which is not seen in the SED. A conservative upper limit on the fractional luminosity of cold dust around HD $15407 \mathrm{~A}$ is set as $L_{\text {dust }} / L_{*} \lesssim 2 \times 10^{-6}$, from a maximum thermal emission of additional blackbody of $50 \mathrm{~K}$ over the MIR excess emission allowed within the uncertainties of Herschel/PACS photometries. Although the $160 \mu \mathrm{m}$ photometry of Herschel/PACS lies slightly above the estimated continuum level, the uncertainty is large, and thus the presence of very cold dust $(\lesssim 30 \mathrm{~K})$ is not confirmed at present.

\subsection{Mass of Warm Debris around HD $1504^{7} 7 \mathrm{~A}$}

Fuiiwara et al. (2012a) estimated the mass of fine warm dust around HD 15407A as $7 \times 10^{17} \mathrm{~kg} \sim 10^{-7} M_{\oplus}$ 
from the dust model fitted to the band features seen in the Spitzer/IRS spectrum at 5-35 $\mu \mathrm{m}$. This value does not include the mass of the blackbody dust, which consists of large ( $\gtrsim 10 \mu \mathrm{m}$ in size) grains and rubbles.

Assuming that the continuum component of the observed excess emission at MIR and FIR wavelengths is attributable to blackbody grains of $505 \mathrm{~K}$ at $R_{\text {dust }}=$ $0.6 \mathrm{AU}$ as suggested by Fuijwara et al. (2012a) and this work and that its flux density is proportional to the sum of geometrical cross sections of the dust with the grain size distribution of $n(a) \propto a^{-3.5}$, we could estimate the total mass of large debris dust as

$$
\begin{aligned}
\frac{M_{\text {total }}}{M_{\oplus}}=2.7 \times 10^{-4}\left(\frac{L_{\text {dust }}}{L_{*}}\right) & \left(\frac{\rho}{2.5 \mathrm{~g} \mathrm{~cm}^{3}}\right)\left(\frac{a_{\mathrm{min}}}{\mu \mathrm{m}}\right)^{\frac{1}{2}} \\
& \times\left(\frac{a_{\mathrm{max}}}{\mu \mathrm{m}}\right)^{\frac{1}{2}}\left(\frac{R_{\text {dust }}}{\mathrm{AU}}\right)^{2},
\end{aligned}
$$

(Hillenbrand et al. 2008), where $\rho, a_{\min }$, and $a_{\max }$ are the specific density and the minimum and maximum grain size of dust, respectively. Adopting $L_{\mathrm{dust}} / L_{*}=0.005$ for the continuum excess emission from warm dust around HD $15407 \mathrm{~A}, \rho=2.5 \mathrm{~g} \mathrm{~cm}^{-3}$ of silicate, $a_{\min }=10 \mu \mathrm{m}$ as the smallest size of grain with featureless spectrum in the $\mathrm{MIR}$, and $R_{\text {dust }}=0.6 \mathrm{AU}$, the total mass is calculated as

$$
\frac{M_{\mathrm{total}}}{M_{\oplus}}=1.5 \times 10^{-6}\left(\frac{a_{\mathrm{max}}}{\mu \mathrm{m}}\right)^{\frac{1}{2}} .
$$

The maximum size $a_{\max }$ is not known though. The total mass of the warm debris dust around HD 15407A is estimated as $\sim 5 \times 10^{-5} M_{\oplus} \sim 3 \times 10^{20} \mathrm{~kg}$ assuming $a_{\max }=1 \mathrm{~mm}$. Assuming $a_{\max }=1 \mathrm{~km}$, the total mass is estimated as $\sim 0.05 M_{\oplus} \sim 3 \times 10^{23} \mathrm{~kg}$, corresponding to a few lunar masses or about a half of Mars mass.

An upper limit on the mass of cold dust $(\sim 50 \mathrm{~K}$ at $R_{\text {dust }}=60 \mathrm{AU}$ ) around HD 15047A could also be estimated in the same manner from the upper limit on the fractional luminosity $L_{\text {dust }} / L_{*} \lesssim 2 \times 10^{-6}$ set by the present work. Assuming that the same grain size distribution $\left(n(a), a_{\min }, a_{\max }\right)$ as that of warm dust, an upper limit on the mass of cold dust is given as just a few times of the mass of warm dust as estimated above.

\subsection{NIR Excess}

As mentioned in the previous subsection, no debris material colder than $\sim 500 \mathrm{~K}$ is suggested by the MIRFIR SED of HD 15407A. On the other hand, photometry at $4.6 \mu \mathrm{m}$ in the WISE W2-band shows excess over the estimated continuum component of blackbody (Figure 1), suggesting the presence of another component in addition to the warm dust components examined by Fuiiwara et al. (2012a). Spitzer/IRS observations of HD 15407A show that the residual spectrum subtracted by the best-fit model of the $5-35 \mu \mathrm{m}$ excess increases toward $\lambda \lesssim 5 \mu \mathrm{m}$. Weak excess emission at $3.4 \mu \mathrm{m}$ over the continuum component is also seen in the WISE W1-band data. Figure 2 plots the residual flux densities of excess emission at 3-7 $\mu \mathrm{m}$ subtracted by the warm (500-600 K) dust components derived in Fujiwara et al. (2012a). Although the residual flux densities seen around 3-6 $\mu \mathrm{m}$ might be attributable to hotter dust ( $\gtrsim 1000 \mathrm{~K})$ in the vicinity of the star, the rise of the residual toward $\sim 4.6 \mu \mathrm{m}$ from its both sides seems too steep, suggesting the possible presence of a band feature attributable to circumstellar gas. A possible carrier of the NIR excess is the first overtone mode of $\mathrm{SiO}$ gas around $4 \mu \mathrm{m}$, which might be related to collisional event of rocky bodies (see Section (4).

\section{DISCUSSION}

MIR observations of HD 15407A by AKARI/IRC and Spitzer/IRS revealed the presence of a large amount of warm dust $(500-600 \mathrm{~K})$ at the distance of $\lesssim 1$ AU from the central star. FIR observations of the star by Herschel/PACS and AKARI/FIS in this work suggest remarkable results - cold dust at $\gtrsim 10 \mathrm{AU}$ is absent and all of the measured $60-100 \mu \mathrm{m}$ emission comes from the same population of dust grains that produce the bright MIR emission seen by AKARI/IRC and Spitzer/IRS. The results are contrary to a previous prediction by Olofsson et al. (2012) who suggested the presence of a population of cool dust around the star based on an MIR spectral model. NIR-MIR observations by WISE also suggest the possible presence of hotter dust or gas component. In summary, the HD 15407A system possesses abundant warm dust at $\lesssim 1 \mathrm{AU}$ and no cold dust at $\gtrsim 10 \mathrm{AU}$, which suggests that the system is quite different from most of the known debris disks with cold dust mainly discovered by $\operatorname{IRAS}$ (Rhee et al. 2007). So far about 10 bright warm debris disks around solar-type stars are known. Among those, HD 15407A seems similar to the G-star BD +20 307, which possesses dust within $1 \mathrm{AU}$ and no cold dust in the further region (Weinberger et al. 2011).

An interesting issue of the debris disk around HD 15407A is its large fractional luminosity, which is estimated as $\sim 0.005$ solely from MIR observations by Fujiwara et al. (2012a). This value is still secure even if the flux densities in the FIR are taken into account. Although the estimated age ranges from $80 \mathrm{Myr}$ to $2 \mathrm{Gyr}$, the fractional luminosity of the disk is exceptionally $\left(\times 10^{4}-10^{5}\right)$ larger than those predicted by steady-state models of planetesimal collisions for the suggested age range $\left(10^{-6}-10^{-8}\right.$; Wyatt et al. 2007). Transient events are thus suggested to be responsible for the large amount of debris dust around HD 15407A.

As the formation mechanism of warm debris disk around the $\sim 1$ Gyr old main-sequence star $\eta$ Corvi, an LHB-like event is suggested by Lisse et al. (2012). Submillimeter observations by James Clerk Maxwell Telescope detect cold debris belt at $\sim 150 \mathrm{AU}$ around the star (Wyatt et al. 2005), which might form from planetesimals in the outer region (analog of Kuipe Belt objects). The MIR spectrum of the star suggests the presence of a large amount of warm debris dust, which might be produced at a few AU through collision(s) of an icy Kuiper Belt body or bodies falling from the outer region of the system (Lisse et al. 2012). In the scheme of an LHB event, the presence of a large amount of planetesimals in the outer region is required as impactors in the inner region. Self-grinding of the planetesimals should produce abundant small dust grains with low temperatures, which produce thermal emission at FIR wavelengths, as seen toward $\eta$ Corvi. $N$-body simulations of the LHB in the solar system by Booth et al. (2009) based on the Nice 
model (Gomes et al. 2005) suggest that the mass surface density of debris at $\sim 20-30$ AU from the Sun would be $\sim 10$ times larger than that at a few AU during the LHB. Therefore, the absence of cold dust around HD 15047A does not support an LHB-like event as a source of the warm dust around HD 15407A.

Catastrophic collisions of two rocky, planetary-scale bodies in the terrestrial zone, which are an analog of the giant impact in the solar system, is suggested as a most likely source of warm debris around BD +20 307 (Weinberger et al. 2011). This giant-impact-like event might be applicable to the origin of the disk with warm debris and no cold material around HD 15407A, since a giant-impact-like event does not require a reservoir of planetesimals in the outer region. Lisse et al. (2009) conclude that the detection of silica dust and $\mathrm{SiO}$ gas features toward HD 172555 suggests a hypervelocity impact as the origin of the debris disk. Detection of abundant silica dust around HD 15407A would be harmonic with the possible mechanism of debris akin to that of HD 172555, but the presence of $\mathrm{SiO}$ gas around $\mathrm{HD}$ 15407A is not confirmed in its Spitzer/IRS spectrum (Fujiwara et al. 2012a). Spectroscopic observations at $3-5 \mu \mathrm{m}$ are needed to search for a hint of the first overtone feature of $\mathrm{SiO}$ gas.

A giant impact is a probable hypothesis for the Moon formation around the Earth (Canup 2004) and is predicted to have been common during the final stage of terrestrial planet formation in extra-solar systems (Kenvon \& Bromlev 2006). A large amount of debris should be ejected in a giant impact. A recent theoretical study of the evocation of debris created in the Moonforming giant impact (Jackson \& Wyatt 2012) suggests that a giant impact generates a debris ring around the Earth orbit and that the fractional luminosity of the debris ring depends on the size of the largest fragment as well as the time after the impact. According to Jackson \& Wyatt (2012), a fractional luminosity larger than 0.005 would be achieved until $100 \mathrm{yr}$ after the impact when the size of the largest fragment is $1-10^{3} \mathrm{~m}$ and would not be achieved when the size of largest fragment is $>10 \mathrm{~km}$. Assuming $100 \mathrm{yr}$ as the lifetime of a giant-impact-generated bright debris disk, the probability to detect such an event is $\sim 10^{-6}$ and $10^{-7}$ for a star with age of $100 \mathrm{Myr}$ and $1 \mathrm{Gyr}$, respectively. Even if we consider that the formation of an Earth-like planet requires around 10 giant impacts (e.g. Kenyon \& Bromley 2006), the probability increases only by one order of magnitude. HD $15407 \mathrm{~A}$ is found as a possible giant-impact star among $\sim 600$ FGK dwarf stars in the $A K A R I$ survey (Fujiwara et al. 2012b) and the apparent probability is $\sim 10^{-3}$, which is much larger than the value estimated above. The discrepancy might be due to an observational bias since MIR-luminous sources are easy to be found from the survey. A complete census of debris disks in a larger volume space by WISE may fill the gap.

It should be noted that HD 15407A is in a possible binary system with the K2V star HD 15407B with the separation of $21 . ! 2$ (projected distance of $1170 \mathrm{AU}$ ) and HD 15407B might disturb the circumstellar material around HD 15407A dynamically. The study of the stability zone in a binary system by Holman \& Wiegert (1999) suggests that materials at $<400$ and $<150 \mathrm{AU}$ from HD 15407A are dynamically stable when the eccentricity of the system is 0.0 and 0.5 , respectively. Thus the absence of cold materials at $\sim 10-60$ AU around the star is not due to the dynamical effect in the binary system.

This research is based on observations with Herschel, AKARI, Spitzer, WISE, IRAS, 2MASS, and Subaru Telescope. We thank C.M. Lisse, M. Shirahata, and the anonymous referee for their useful comments. This work was supported by KAKENHI (23103002).

Facilities: Herschel (ESA), AKARI (ISAS/JAXA), Spitzer (NASA), WISE (NASA), Subaru (NAOJ)

\section{REFERENCES}

Backman, D. E., \& Paresce, F. 1993, Protostars and Planets III, 1253

Beichman, C. A., Neugebauer, G., Habing, H. J., Clegg, P. E., \& Chester, T. J. 1988, Infrared astronomical satellite (IRAS) catalogs and atlases. Volume 1: Explanatory supplement, 1,

Booth, M., Wyatt, M. C., Morbidelli, A., Moro-Martín, A., \& Levison, H. F. 2009, MNRAS, 399, 385

Canup, R. M. 2004, ARA\&A, 42, 441

Cutri, R. M., Skrutskie, M. F., van Dyk, S., et al. 2003, "The IRSA 2MASS All-Sky Point Source Catalog, NASA/IPAC Infrared Science Archive.

Cutri, R. M., Wright, E. L., Conrow, T., et al. 2012, Explanatory Supplement to the WISE All-Sky Data Release Products, 1

Fujiwara, H., Onaka, T., Ishihara, D., et al. 2010, ApJ, 714, L152

Fujiwara, H., Onaka, T., Yamashita, T., et al. 2012a, ApJ, 749, L29

Fujiwara, H., Ishihara, D., Onaka T., et al. 2012b, submitted to A\&A

Gomes, R., Levison, H. F., Tsiganis, K., \& Morbidelli, A. 2005, Nature, 435, 466

Hillenbrand, L. A., Carpenter, J. M., Kim, J. S., et al. 2008, ApJ, 677,630

Holman, M. J., \& Wiegert, P. A. 1999, AJ, 117, 621

Holmberg, J., Nordström, B., \& Andersen, J. 2009, A\&A, 501, 941

Ishihara, D., Onaka, T., Kataza, H., et al. 2010, A\&A, 514, A1

Jackson, A. P., \& Wyatt, M. C. 2012, MNRAS, 425, 657

Kawada, M., Baba, H., Barthel, P. D., et al. 2007, PASJ, 59, 389

Kenyon, S. J., \& Bromley, B. C. 2006, AJ, 131, 1837

Kurucz, R. L. 1992, The Stellar Populations of Galaxies, 149, 225

Lisse, C. M., Chen, C. H., Wyatt, M. C., Morlok, A., Song, I., Bryden, G., \& Sheehan, P. 2009, ApJ, 701, 2019

Lisse, C. M., Wyatt, M. C., Chen, C. H., et al. 2012, ApJ, 747, 93

Melis, C., Zuckerman, B., Rhee, J. H., \& Song, I. 2010, ApJ, 717, L57

Olofsson, J., Juhász, A., Henning, T., et al. 2012, A\&A, 542, A90

Oudmaijer, R. D., van der Veen, W. E. C. J., Waters,

L. B. F. M., Trams, N. R., Waelkens, C., \& Engelsman, E. 1992, A\&AS, 96, 625

PACS Observer's Manual 2011

http://herschel.esac.esa.int/Docs/PACS/pdf/pacs_om.pdf

Pilbratt, G. L., Riedinger, J. R., Passvogel, T., et al. 2010, A\&A, 518, L1

Poglitsch, A., Waelkens, C., Geis, N., et al. 2010, A\&A, 518, L2

Rhee, J. H., Song, I., Zuckerman, B., \& McElwain, M. 2007, ApJ, 660,1556

Shirahata, M., Matsuura, S., Hasegawa, S., et al. 2009, PASJ, 61, 737

van Leeuwen, F. 2007, Astrophysics and Space Science Library, 350

Weinberger, A. J., Becklin, E. E., Song, I., \& Zuckerman, B. 2011, ApJ, 726, 72

Wyatt, M. C., Greaves, J. S., Dent, W. R. F., \& Coulson, I. M. 2005, ApJ, 620, 492

Wyatt, M. C., Smith, R., Greaves, J. S., Beichman, C. A., Bryden, G., \& Lisse, C. M. 2007, ApJ, 658, 569 
Table 1

Infrared Photometry of HD 15407A

\begin{tabular}{lrrr}
\hline Instrument (Filter) & $\begin{array}{r}\text { Wavelength } \\
(\mu \mathrm{m})\end{array}$ & $\begin{array}{r}\text { Flux Density } \\
(\mathrm{Jy})\end{array}$ & $\begin{array}{r}\text { Photosphere } \\
(\mathrm{Jy})\end{array}$ \\
\hline 2MASS (J) & 1.22 & $6.017 \pm 0.181$ & $\ldots$ \\
2MASS $(H)$ & 1.65 & $4.352 \pm 0.136$ & $\ldots$ \\
2MASS (KS) & 2.16 & $3.105 \pm 0.062$ & $\ldots$ \\
WISE $(W 1)$ & 3.4 & $1.729 \pm 0.093$ & 1.53 \\
WISE (W2) & 4.6 & $1.381 \pm 0.040$ & 0.88 \\
WISE (W3) & 12 & $0.701 \pm 0.010$ & 0.12 \\
WISE (W4) & 22 & $0.405 \pm 0.009$ & 0.04 \\
AKARI/IRC (S9W) & 9 & $0.960 \pm 0.030$ & 0.22 \\
AKARI/IRC (L18W) & 18 & $0.500 \pm 0.020$ & 0.06 \\
Subaru/COMICS (N8.8) & 8.8 & $0.904 \pm 0.090$ & 0.26 \\
Subaru/COMICS (N11.7) & 11.7 & $0.644 \pm 0.064$ & 0.15 \\
Subaru/COMICS (Q18.8) & 18.8 & $0.486 \pm 0.073$ & 0.06 \\
IRAS & 12 & $1.050 \pm 0.060$ & 0.12 \\
IRAS & 25 & $0.430 \pm 0.030$ & 0.03 \\
IRAS & 60 & $<0.400$ & 0.005 \\
IRAS & 100 & $<1.140$ & 0.002 \\
AKARI/FIS (WIDE-S) & 90 & $0.077 \pm 0.061$ & 0.003 \\
Herschel/PACS (Blue) & 70 & $0.050 \pm 0.011$ & 0.004 \\
Herschel/PACS (Green) & 100 & $0.022 \pm 0.005$ & 0.002 \\
Herschel/PACS (Red) & 160 & $0.025 \pm 0.014$ & 0.0008 \\
\hline
\end{tabular}




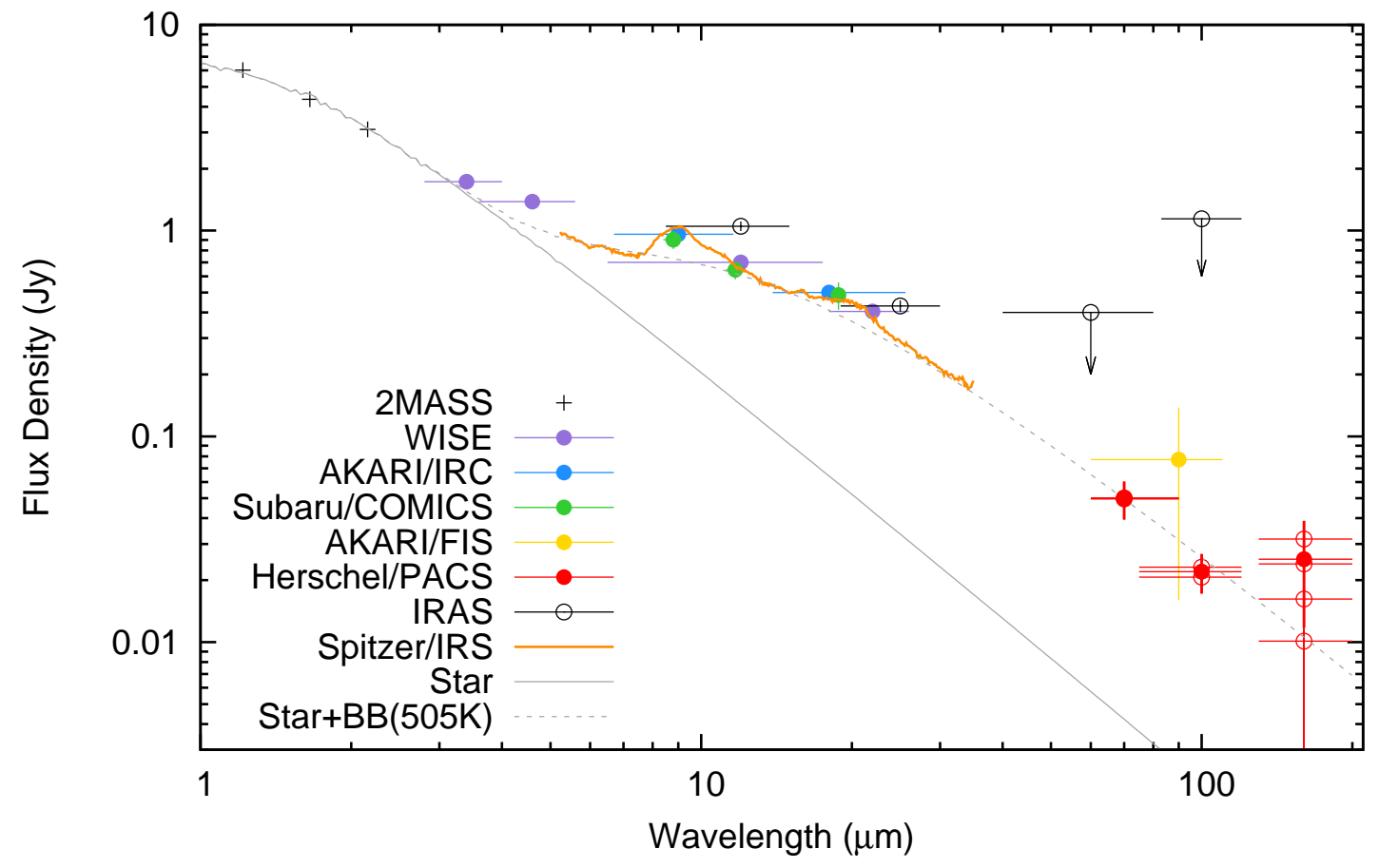

Figure 1. NIR-FIR SED of HD 15407A. The black crosses indicate the 2MASS photometry, which determines the photospheric contribution of the star (Kurucz 1992) shown as the gray solid line. The purple, blue, green, yellow filled circles, and black open circles indicate the photometric data of WISE, AKARI/IRC, Subaru/COMICS, and AKARI/FIS, and IRAS, respectively. The red open and filled circles indicate the photometric measurements from individual images taken with Herschel/PACS and their weighted-average of photometry for each band, respectively. All the flux densities are not color-corrected. The vertical and horizontal bars of the photometric data show the flux density errors and the bandwidths, respectively. The orange solid and gray dashed lines indicate the Spitzer/IRS spectrum and the estimated continuum level of the excess emission, respectively, taken from Fuijwara et al. (2012a).

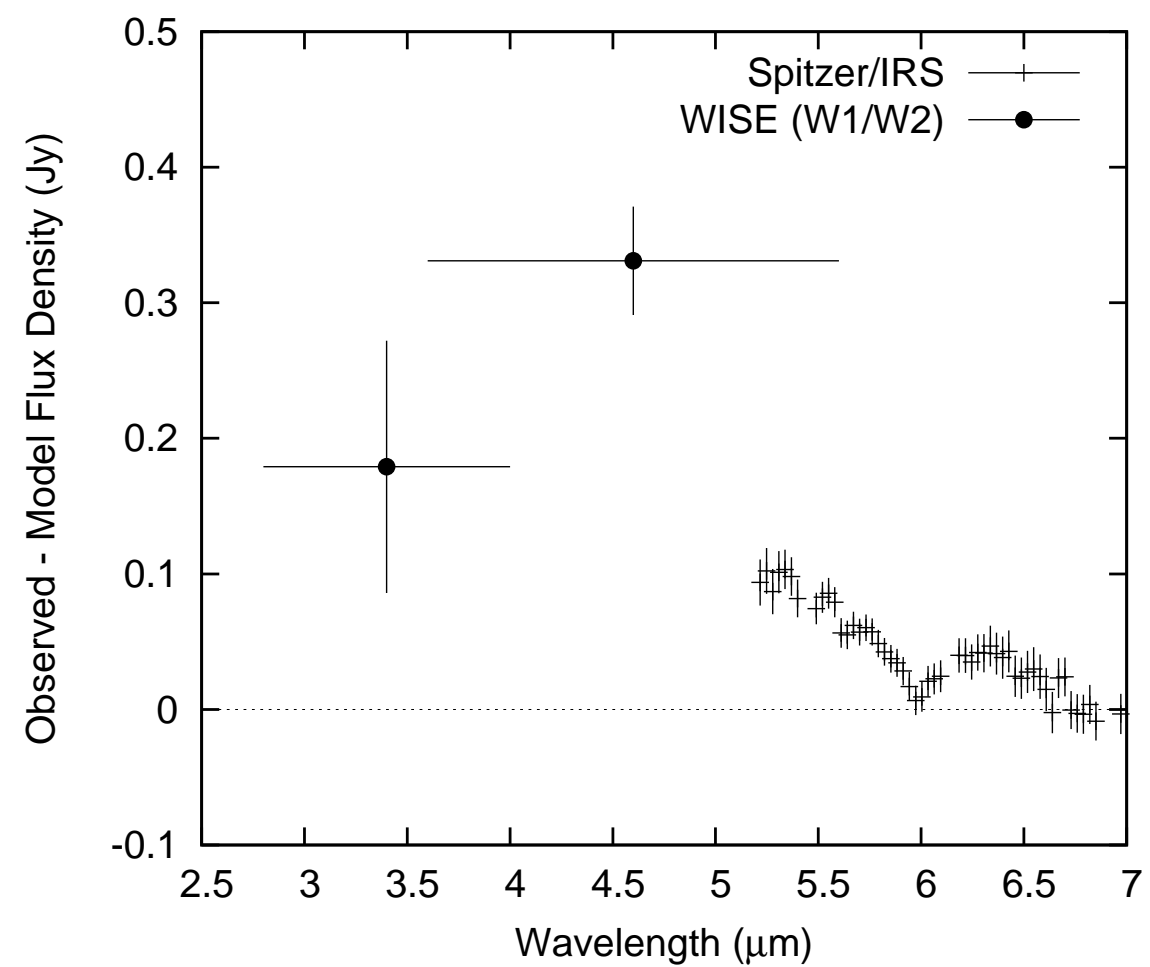

Figure 2. NIR-MIR residual flux densities of HD 15407A subtracted by the model fitted to the Spitzer/IRS spectrum at 5-35 $\mu$ m. The crosses and filled circles indicate data from Spitzer/IRS and WISE, respectively. The vertical and horizontal bars of the photometric data show the flux density errors and the bandwidths, respectively. 